\title{
Definitive and adjuvant radiotherapy for sinonasal squamous cell carcinomas: a single institutional experience
}

\author{
Sumerya Duru Birgi, Mark Teo, Karen E. Dyker, Mehmet Sen and Robin J D Prestwich*
}

\begin{abstract}
Background: The aim of this study was to evaluate the disease outcomes of patients treated with definitive and adjuvant radiotherapy for squamous cell carcinomas of the nasal cavity and paranasal sinuses in a single institution.

Methods: Between 2007-2012 patients were retrospectively identified from electronic databases who had undergone surgery and adjuvant radiotherapy or definitive radiotherapy for sinonasal squamous cell carcinomas with curative intent.

Results: Fourty three patients with sinonasal squamous cell carcinoma were identified (22 nasal cavity, 21 paranasal sinuses). 31/43 (72 \%) had T3 or T4 disease; nodal stage was N0 in 38, N1 in 4, Na/b in 0 and N2c in 1 patient. Median age was 67 years (range 41-86). 18 (42\%) received definitive and 25 (58 \%) adjuvant radiotherapy. Radiotherapy was delivered using either conventional radiotherapy $(n=39)$ or intensity modulated radiotherapy $(n=4)$. Elective neck radiotherapy was delivered to two patients. Chemotherapy was delivered to 6/43 (14 \%) of patients. Two-year local control, regional control, distant metastases free survival, progression free survival, cause specific survival and overall survival were $81 \%, 90 \%, 95 \%, 71 \%, 84 \%$ and $80 \%$ respectively. There was no significant difference in outcome comparing patients who underwent surgery and adjuvant radiotherapy with patients receiving definitive radiotherapy ( 2 year locoregional disease free survival $75 \%$ and $70 \%$ respectively, $p=0.98$ ). Pooly differentiated tumours were significantly associated with inferior disease outcomes. Local, regional, combined local and regional, and distant failure occurred in $7(16 \%), 3(7 \%), 1$ (2\%) and $2(5 \%)$ of patients; all 3 regional recurrences were in patients with nasal cavity squamous cell carcinomas who had not undergone elective neck treatment.

Conclusions: Definitive or adjuvant radiotherapy provides an effective treatment for sinonasal malignancies. The main pattern of failure remains local, suggesting the need for investigation of intensified local therapy. Whilst remaining uncommon, the cases of regional failure mean that the merits of elective lymph node treatment should be considered on an individual basis.
\end{abstract}

\section{Introduction}

Sinonasal malignancies are rare [1] and include a wide range of histopathological cell types [2,3]. The anatomical structure of the sinuses allows asymptomatic growth until local structures/organs are invaded; most patients therefore present with locally advanced disease [4]. Determining optimal management continues to be challenging due to the rarity of the disease and proximity to multiple

\footnotetext{
* Correspondence: Robin.Prestwich@nhs.net

This work was presented in part at the 5 th International Conference on Innovative Approaches in Head and Neck Oncology (ICHNO) 2015. Department Of Clinical Oncology, St. James's Institute of Oncology, Level 4, Bexley Wing, Beckett Street, Leeds, West Yorkshire LS9 7TF, UK
}

critical structures including the optic nerves, chiasm, eyes and brainstem. Management choices have largely been informed by single institution retrospective series. Treatment approaches have generally involved surgery followed by adjuvant radiotherapy, or definitive radiotherapy for patients for whom complete surgical excision is unlikely, co-morbidity or patient preference $[4,5]$.

Controversies remain in the management of sinonasal squamous cell carcinomas. Local failure is the major pattern of relapse and has guided treatment approaches [6]. However, there is variation in practice regarding the necessity for elective treatment of a clinically/radiologically N0 neck $[6,7]$. In addition, by contrast with 
more common cancers sites within the head and neck region, there is not a strong evidence based for the role of chemotherapy; this at least partly relates to the rarity of the disease. The use of chemotherapy has been generally at clinician discretion in several series $[2,5,8]$

In this study, we report the disease outcomes and patterns of failure for patients with sinonasal squamous cell carcinomas treated with radiotherapy in the postoperative or definitive setting.

\section{Materials and methods \\ Study design}

A retrospective study was performed using patient records, radiotherapy treatment plans and diagnostic imaging on 43 patients who had been treated with either definitive or adjuvant radiotherapy between 2007-2012 for sinonasal squamous cell cancers. Patients were excluded if radiotherapy was delivered with palliative intent. Patients were staged according to the American Joint Committee on Cancer 7th Edition [9]. Clinical and radiological staging was used for patients treated nonsurgically, whilst pathological staging was used for patients who had undergone surgery.

\section{Treatment}

\section{Surgery}

All patients underwent evaluation in a Head and Neck multidisciplinary team to evaluate the feasibility/ appropriateness of surgical resection. The type of surgery depended upon primary site, extent of disease, cosmetic considerations and discretion of the surgical team; surgery was always aimed at obtaining a gross total resection.

\section{Radiotherapy}

Three patients with a nasal vestibule cancer were treated with a direct electron field. 39/43 (91 \%) of patients were treated with a 3D-conformal photon technique and toward the end of the study period six patients $(12 \%)$ were treated with intensity modulated radiothearapy (IMRT). Patients were immobilised with a Perspex mask with a neutral neck position. A CT scan with 2-5 mm slices was acquired. MRI co-registration was not available during the study period. A mouthbite was routinely used to minimise the radiation dose to the lower part of the oral cavity. A compartmental approach to target volume delineation was adopted. For patients receiving definitive radiotherapy a gross tumour volume (GTV) was outlined as primary tumour and clinically and/or radiologically involved lymph nodes. A primary tumour clinical target volume (CTV) was created to include at least GTV $+10 \mathrm{~mm}$ and modified to include the whole involved anatomical compartment or sinus ie. all invaded or partly invaded sinuses, modified to anatomical boundaries to exclude air and/or bone without evidence of invasion. For patients who had undergone macroscopically complete surgical resection of the tumour, the same compartmental approach was adopted to delineate the CTV encompassing the resection cavity. Elective treatment of the neck was not routinely performed for clinically node negative disease; neck treatment was delivered for $\mathrm{N}+$ neck disease. Planning target volumes (PTV) were generated by an auto-expansion of $4 \mathrm{~mm}$ to CTV structures.

Organs at risk routinely delineated on the planning CT scan included: optic chiasm, brainstem, optic nerves, spinal cord.

Radiotherapy was delivered five fractions per week. The prescription dose was at the discretion of the treating clinician; standard adjuvant doses were 60-66Gy in 30-33 fractions and definitive doses included a hypofractionated regimen of 55Gy in 20 fractions in the early part of the study period, and conventionally fractionated 66-70Gy in 33-35 fractions in the latter part of the study. Standard dose constraints during this study period were: spinal cord $<48 \mathrm{~Gy}$, optic nerves, brainstem and chiasm $<54$ y, lens as low as achievable.

The 3D-conformal technique was based on either a three-field technique with a heavily weighted anterior portal with two lateral wedged portals or an anterior wedged pair technique depending upon the target volume. IMRT was delivered with a 5-7 angle step and shoot IMRT technique.

All photon treatment was delivered with $6 \mathrm{MV}$ photons. Treatment was planned to provide adequate coverage of the target volumes according to ICRU-62 guidelines [10].

\section{Chemotherapy}

Chemotherapy was employed upon clinician discretion, based upon tumour histology and high risk features. Concurrent chemotherapy was with cisplatin $100 \mathrm{mg} / \mathrm{m}^{2}$ days 1 and 29 . Induction chemotherapy regimens included cisplatin $80 \mathrm{mg} / \mathrm{m}^{2}$ day 1 and 5 fluorouracil (5FU) $800 \mathrm{mg} / \mathrm{m}^{2}$ days $2-5$, three weekly.

\section{Follow up schedule}

Patients were seen at least once per week during radiotherapy treatment. For patients receiving definitive radiotherapy, response assessment imaging with CT and/or MRI was routinely performed 3 months post-treatment. Patients were followed up with physical examination every 6-8 weeks in the first year after treatment, every 3 months for an additional 2 years, and every 6 monthly until discharge at 5 years. Suspected recurrence was detected by clinical examination and radiological examination and biopsy if appropriate.

\section{Statistical analysis}

Statistical analysis was performed using SPSS version 16 (IBM, USA), STATA version 10 (Statacorp, USA), and 
Table 1 Patient and tumour characteristics

\begin{tabular}{|c|c|c|}
\hline Characteristics & Number $(n=43)$ & Percent \\
\hline \multicolumn{3}{|l|}{ Gender } \\
\hline Male & 25 & 60 \\
\hline Female & 18 & 40 \\
\hline \multicolumn{3}{|l|}{ Tobacco use at diagnosis } \\
\hline Ex smoker & 8 & 19 \\
\hline Current smoker & 15 & 35 \\
\hline No smoker & 7 & 16 \\
\hline Not documented & 13 & 30 \\
\hline \multicolumn{3}{|l|}{ Tumour localisation } \\
\hline Maxillary sinus & 20 & 47 \\
\hline Ethmoid sinus & 1 & 2 \\
\hline Nasal cavity & 22 & 51 \\
\hline Nasal vestibule & 10 & 23 \\
\hline Nasal septum & 3 & 7 \\
\hline Floor and lateral wall & 9 & 21 \\
\hline \multicolumn{3}{|l|}{ T stage } \\
\hline TO & 0 & 0 \\
\hline $\mathrm{T} 1$ & 6 & 14 \\
\hline $\mathrm{T} 2$ & 6 & 14 \\
\hline T3 & 2 & 5 \\
\hline T4a & 23 & 53 \\
\hline T4b & 6 & 14 \\
\hline \multicolumn{3}{|l|}{ N stage } \\
\hline NO & 38 & 88 \\
\hline N1 & 4 & 9 \\
\hline $\mathrm{N} 2 \mathrm{a}$ & 0 & 0 \\
\hline $\mathrm{N} 2 \mathrm{~b}$ & 0 & 0 \\
\hline $\mathrm{N} 2 \mathrm{C}$ & 1 & 3 \\
\hline \multicolumn{3}{|l|}{ Stage group (AJCC 2010) } \\
\hline । & 6 & 14 \\
\hline$\|$ & 6 & 14 \\
\hline III & 2 & 5 \\
\hline IVa & 23 & 53 \\
\hline $\mathrm{IVb}$ & 6 & 14 \\
\hline \multicolumn{3}{|l|}{ Histopathology } \\
\hline SCC & 43 & 100 \\
\hline \multicolumn{3}{|c|}{ Histopathological differentiation } \\
\hline Well differentiated & 7 & 16 \\
\hline Moderately differentiated & 18 & 40 \\
\hline Poorly differentiated & 10 & 23 \\
\hline Undifferentiated & 3 & 7 \\
\hline Not documented & 6 & 14 \\
\hline
\end{tabular}

Table 1 Patient and tumour characteristics (Continued)

\begin{tabular}{lll}
\hline $\begin{array}{l}\text { Nodal involvement } \\
\text { Yes }\end{array}$ & 3 & 7 \\
No & 40 & 93 \\
Nodal ECE & & \\
Yes & 1 & 20 \\
No & 4 & 80 \\
Tumor invasion & & \\
Cranial & 2 & 5 \\
Orbital & 2 & 5 \\
Skin & 7 & 16 \\
\hline
\end{tabular}

Prism version 6 (Graphpad, USA). Survival and recurrence outcomes were calculated from the date of their curative treatment (i.e. date of curative surgery, date of first fraction of radiotherapy). The following endpoints were used for assessment: local control rate, regional control rate, locoregional disease free survival (LRDFS), distant metastases free survival (DMFS), progression free survival (PFS), cause specific survival (CSS) and overall survival (OS). For comparison of surgery and adjuvant radiotherapy versus definitive radiotherapy, univariate log-rank survival analysis was performed for each outcome and hazard ratios (HR) and $95 \%$ confidence intervals calculated.

\section{Results}

Fourty three patients with sinonasal squamous cell carcinomas were identified who had received definitive or adjuvant radiotherapy. Patient and disease characteristics are summarised in Table 1. Median follow up was 32 (range 4-102) months. Median age was 67 years (range 41-86). 35/52 (67 \%) had T4a or T4b disease.

Treatment details are summarised in Table 2. 25/43 $(58 \%)$ of patients had undergone surgery, with a positive resection margin $(<1 \mathrm{~mm})$ reported in $13 / 25(52 \%)$ and close $(<5 \mathrm{~mm})$ margins in 5/25 (20\%). 36/43 (84\%) of patients received radiotherapy via a 3D-conformal technique. Neck radiotherapy was delivered to 3 patients; in one case neck radiotherapy followed neck dissections for $\mathrm{N}+$ disease and in two cases elective neck radiotherapy was delivered to the ipsilateral neck along with treatment of the primary site following surgery for T4N0 squamous cell carcinomas of the maxillary sinus. Chemotherapy was delivered to a total of 6/43 (14 \%) of patients. Comparing patients who underwent surgery and adjuvant radiotherapy and patients who received definitive radiotherapy, there were significantly more patients undergoing surgery with T4a disease $(p<0.001)$ and a non-significant trend for more patients with maxillary tumours to undergo surgery $(p=0.06) .12 / 18(67 \%)$ patients who received 
Table 2 Treatment details

\begin{tabular}{|c|c|c|}
\hline & $\begin{array}{l}\text { Number }(n)(n=43 \text { unless } \\
\text { stated otherwise) }\end{array}$ & Percentage (\%) \\
\hline Surgery & $n=25$ & 58 \\
\hline Open & 23 & 92 \\
\hline Endoscopy & 2 & 8 \\
\hline Unilateral neck dissection & 12 & 48 \\
\hline Bilateral neck dissection & 2 & 8 \\
\hline Margin status & $n=25$ & \\
\hline Positive & 13 & 52 \\
\hline Close $(<5 \mathrm{~mm})$ & 5 & 20 \\
\hline Negative ( $\geq 5 \mathrm{~mm}$ ) & 6 & 24 \\
\hline Unknown & 1 & 4 \\
\hline Perineural invasion & $n=25$ & \\
\hline Yes & 5 & 20 \\
\hline No & 10 & 40 \\
\hline Not reported & 10 & 40 \\
\hline Lymphovascular invasion & $n=25$ & \\
\hline Yes & 1 & 4 \\
\hline No & 13 & 52 \\
\hline Not reported & 11 & 44 \\
\hline \multicolumn{3}{|l|}{ Radiotherapy } \\
\hline Electron & 3 & 7 \\
\hline 3DCRT & 36 & 84 \\
\hline IMRT & 4 & 9 \\
\hline \multicolumn{3}{|l|}{ Radiotherapy } \\
\hline Adjuvant & 25 & 58 \\
\hline Radical & 18 & 42 \\
\hline \multicolumn{3}{|l|}{ Radiotherapy dose } \\
\hline \multicolumn{3}{|l|}{ Radical } \\
\hline $70 \mathrm{~Gy} / 35 \mathrm{fx}$ & 5 & 12 \\
\hline $68 \mathrm{~Gy} / 34 \mathrm{fx}$ & 1 & 2 \\
\hline $66 \mathrm{~Gy} / 33 \mathrm{fx}$ & 2 & 5 \\
\hline $55 \mathrm{~Gy} / 20 \mathrm{fx}$ & 10 & 23 \\
\hline \multicolumn{3}{|l|}{ Adjuvant } \\
\hline $66 \mathrm{~Gy} / 33 \mathrm{fx}$ & 8 & 19 \\
\hline $64 \mathrm{~Gy} / 32 \mathrm{fx}$ & 3 & 7 \\
\hline $62 \mathrm{~Gy} / 31 \mathrm{fx}$ & 1 & 2 \\
\hline $60 \mathrm{~Gy} / 30 \mathrm{fx}$ & 7 & 16 \\
\hline $55 \mathrm{~Gy} / 20 \mathrm{fx}$ & 6 & 14 \\
\hline \multicolumn{3}{|l|}{ Radiotherapy region } \\
\hline Primary & 40 & 93 \\
\hline Primary and ipsilateral neck & 3 & 7 \\
\hline Primary and bilateral neck & 0 & 0 \\
\hline \multicolumn{3}{|l|}{ Chemotherapy } \\
\hline Induction & 3 & 7 \\
\hline Concurrent & 3 & 7 \\
\hline
\end{tabular}

definitive radiotherapy were documented as having achieved a complete clinical and radiological response to treatment.

The 2-year local control rate, regional control rate, DMFS, PFS, CSS and OS were $81 \%, 90 \%, 95 \%, 71 \%$, $84 \%$, and $80 \%$ respectively. 5 -year local control rate, regional control rate, DMFS, PFS, CSS and OS were $76 \%$, $90 \%, 95 \%, 66 \%, 74 \%$, and $71 \%$ respectively. Locoregional control, PFS and OS are illustrated in Fig. 1. Local, regional, combined local and regional, and distant failure occurred in 7 (16\%), 3 (7\%), 1 (2\%) and $2(5 \%)$ of patients. Median time to local recurrence was not reached. There was no significant difference comparing outcomes of patients who underwent radical radiotherapy $(n=18)$ and surgery and adjuvant radiotherapy $(n=25)$ : 2 -year LRDFS was $70 \%$ versus $75 \%$ (non-significant, $p=0.98$ ), and 2 -year CSS was $94 \%$ versus $79 \%$ (non-significant, $p=0.6$ )). Recurrence patterns are summarised in Table 3. Poorly differentiated tumours were significantly associated with increased risk of developing distant metastases (HR 8.2 (95\% CI1.01-66) $P=0.05$ and inferior CSS (HR2.31(95\% CI1.06-5.0, $P=0.04$ ). A total of eight patients experienced local disease recurrence; one of these cases was in combination with regional failure. Primary tumour site involved nasal cavity in 4, ethmoid sinus in 1 and maxillary sinus in 3 patients respectively; disease stages were T4N0 in 6 patients, T1N0 in one patient and T2NO in one patient. Four of these patients had initially undergone surgery and adjuvant radiotherapy and four patients were initially treated with definitive radiotherapy (one with concurrent chemotherapy). The two patients with early stage disease at outset underwent successful surgical salvage of local recurrence; the remaining six patients were managed with palliative approaches.

The four patients who experienced a regional lymph node recurrence had all undergone surgery and adjuvant radiotherapy without elective neck surgery or irradiation for squamous cell carcinoma of the nasal cavity; one was a pT2 and the remaining cases pT4 disease. In these four cases one recurrence occurred with simultaneous local recurrence. The pattern of regional recurrences were in level II lymph nodes in one case, level Ib lymph nodes in two cases and facial lymph nodes in one case. Two patients with regional lymph node recurrence underwent salvage surgery and radiotherapy; one of these patients subsequently developed widespread distant metastases whilst the other remains disease free. One patient was not deemed fit for further treatment, and one patient with synchronous local and regional progression received palliative chemotherapy. There were no cases of regional recurrences in patients treated for paranasal sinus cancers. 

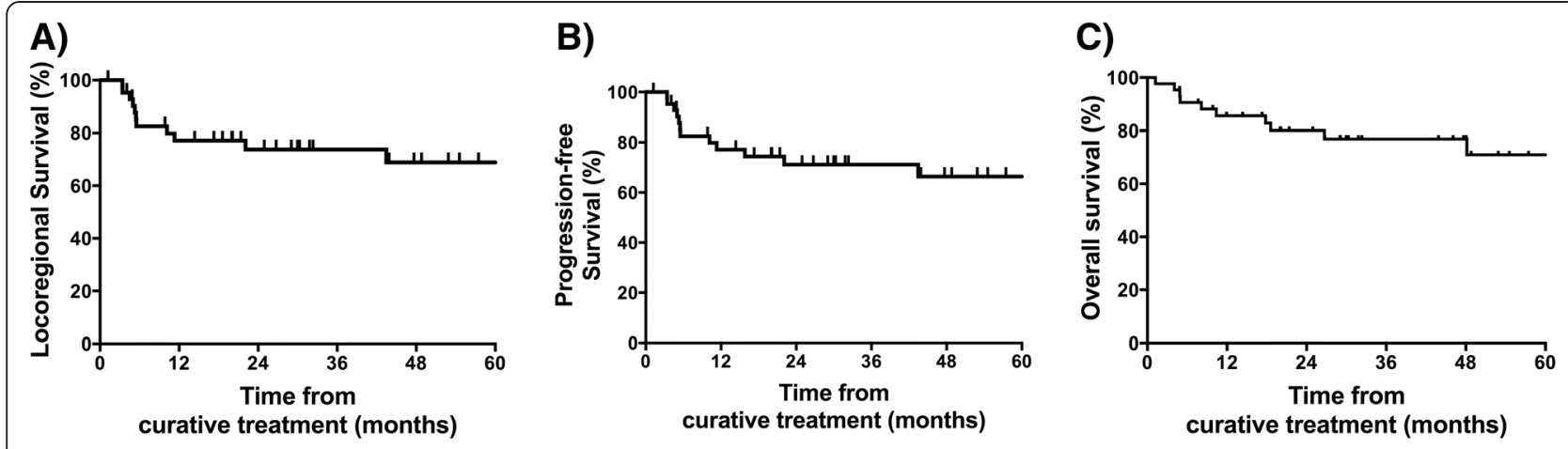

Fig. 1 Kaplan Meier curves showing a locoregional control, b progression free survival and c overall survival

\section{Discussion}

Sinonasal squamous cell carcinomas present a major therapeutic challenge. In the absence of randomised trials, treatment options for sinonasal malignancies are based on retrospective series by institutions; due to the rarity of the disease, data remains limited. Historical outcomes following 2-dimensional radiotherapy are limited in terms of disease outcomes and treatment-related late morbidity [11-13]. The introduction of 3D-conformal radiotherapy facilitated an improvement in disease and toxicity outcomes [14-16]. More recently the advent of IMRT techniques has allowed improved target volume coverage with a minimisation of OAR doses [17]. Reported outcomes in previous studies vary widely; for example, local control rates and overall survival rates are reported between $21-84 \%$ and $9-89 \%$ at 2 to 5 years follow up [2]. Overall outcomes appear to be superior in more recent series. For example, IMRT series have reported 2-year local control rates of $62-76 \%$ [2-5]; 2year overall survival in these modern series ranged from 66-89 \%. Comparison between series is difficult, due the heterogeneity of tumour site, stage, treatment approaches and duration of follow up. In common with other

Table 3 Patterns of disease recurrence

\begin{tabular}{ll}
\hline Recurrence site & Number \\
\hline Local & 4 \\
Radical & 3 \\
Adjuvant & \\
Regional & 0 \\
Radical & 3 \\
Adjuvant & \\
Local and regional & 0 \\
Radical & 1 \\
Adjuvant & \\
Distant metastasis & \\
Radical & 1 \\
Adjuvant & 1 \\
\hline
\end{tabular}

series, our report includes a both a mixture of primary tumour sites including both nasal cavity and paranasal sinuses $[2-5,8,18]$. Broadly, disease outcomes from our series appear favourable compared with many series and similar to those reported recently by other major institutions [2-4]. The proportion of patients in our series with nasal cavity tumours was high $(51 \%)$ compared with other reports $(6-28 \%,[2-5,8,18])$. Although there was no significant difference in outcomes between nasal cavity and non-nasal cavity primary sites, this may have influenced outcomes favourably in our predominantly non-IMRT experience due to the comparative ease by which nasal cavity tumours can be treated compared with other paranasal sinus primaries with 3D-conformal radiotherapy.

The conformality provided by IMRT appears to provide increased levels of local control with very low rates of late toxicity $[3-5,8]$. Recent series reporting outcomes following IMRT for paranasal sinus malignancies have reported no cases of optic neuropathy with limited follow up $[8,19]$. IMRT is now regarded as a standard of care for paranasal sinus cancers [19]. A limitation of our series is that the majority of patients were treated with a 3D-conformal radiotherapy technique; IMRT is now the standard of care within our institution for paranasal sinus tumours.

Patients with a gross tumour resection prior to radiotherapy are associated with favourable outcomes $[18,20,21]$. En bloc removal of macroscopic disease is regarded as the aim of initial treatment. This has been facilitated by ongoing improvements in surgical techniques. There was no significant difference in outcome in patients undergoing definitive radiotherapy and surgery and adjuvant radiotherapy in our series. Although hypothesis generating, these data may be influenced by an imbalance between the groups with an excess of nasal cavity cancers treated non-surgically.

The majority of failures in our series were local recurrences; this is consistent with multiple previous series $[2,3,8]$. This suggests that the focus of improving 
sinonasal tumour outcomes will continue to be improving local treatment outcomes. The excellent results of recent IMRT series suggest that the improved dose conformality leading to less compromise of target coverage may translate into improved local control. Another potential route to improving the efficacy of treatment is the incorporation of chemotherapy. The role of chemotherapy in the treatment of sinonasal malignancies is uncertain. Only $14 \%$ of patients in our series received either induction or concurrent chemotherapy at the discretion of the treating clinician. Similarly in some $[2,22]$ but not all series [3-5], a limited proportion of patients were treated with chemotherapy. An extrapolation from other head and neck cancer sites [23] would suggest that the use of concurrent chemotherapy may be advantageous for squamous cell cancers. Although the delivery of chemotherapy appears feasible as part of treatment for sinonasal malignancies, current reported experiences are unable to provide direct comparative evidence of efficacy. The use of chemotherapy remains unproven for other types of histological diagnosis [24].

Lymph node involvement in sinonasal malignancies is less common compared with several other head and neck tumour sites. However, involvement of lymph nodes at presentation is recognised as a poor prognostic factor for regional and distant disease [8,24, 25], and patients who relapse in regional lymph nodes have an adverse prognosis [8]. In this series only two patient received elective neck irradiation, both for T4N0 squamous cell carcinomas of the maxillary sinus. Four patients developed regional recurrences on follow up; all of these patients had primary cancers of the nasal cavity. The average incidence of neck recurrences in untreated necks following treatment of squamous cell carcinoma of the maxillary sinus is $15 \%$ [6]. The role of elective neck irradiation remains controversial for sinonasal squamous cell carcinomas [3, 6]. The National Comprehensive Cancer Network guidelines recommend elective neck irradiation for T3/T4 squamous cell maxillary carcinomas [26]. A recent meta-analysis has suggested that elective neck irradiation can reduce the rate of nodal recurrence for patients with N0 squamous cell carcinoma of the maxillary sinus [6]. The occurrence of regional nodal recurrence following treatment of nasal cavity cancers suggest that elective nodal radiotherapy could be considered for patients with locally advanced squamous cell carcinoma of the nasal cavity. Factors which need to be considered in evaluating the need for elective neck irradiation for patients on an individual basis include the histological type of malignancy, site, local extent, pattern of potential lymphatic spread and comorbidity.

Distant metastatic failure occurred in 2/43 (5\%) patients; other series including mixed histological diagnoses have reported distant metastases occurring in $18-29 \%$ of patients $[2,4,8]$. These data suggest that although obtaining local control remains a major issue, the potential for distant disease dissemination needs to be considered in designing novel approaches to improve the outcomes of sinonasal malignancies.

One limitation of this series is the absence of robust long term toxicity data. Treatment-related visual impairment may occur with longer term follow up [27]. Toxicity is recorded in an ad-hoc manner without any ophthalmological testing, and as such we are not able to document toxicity in a reliable manner. Importantly, IMRT techniques are now an established method to minimise the risk of optic pathway complications from radiotherapy treatment.

\section{Conclusions}

In summary, definitive or adjuvant radiotherapy provides an effective treatment for sinonasal squamous cell carcinomas. The main pattern of failure remains local, suggesting the need for investigation of intensified local therapy. Whilst remaining uncommon, the cases of regional failure mean that the merits of elective lymph node treatment should be considered on an individual basis.

\section{Abbreviations}

IMRT: Intensity modulated radiotherapy; GTV: Gross tumour volume; CTV: Clinical target volume; PTV: Planning target volume; LRDFS: Locoregional disease free survival; DMFS: Distant metastases free survival; PFS: Progression free survival; CCS: Cause specific survival; OS: Overall survival; HR: Hazard ratio.

\section{Competing interests}

The authors declare that they have no competing interests.

\section{Authors' contributions}

SB carried out data collection, participated in analysis. MT carried out analysis and helped draft manuscript. KD involved in conceiving the study and participated in analysis. MS conceived of the study, participated in analysis. RP coordinated the study, involved in analysis and drafted manuscript. All authors read and approved the final manuscript.

Received: 12 June 2015 Accepted: 3 September 2015

Published online: 17 September 2015

\section{References}

1. Roush GC. Epidemiology of cancer of the nose and paranasal sinuses: current concepts. Head Neck Surg. 1979;2(1):3-11.

2. Wiegner EA, Daly ME, Murphy JD, Abelson J, Chapman $\mathrm{CH}$, Chung M, et al. Intensity-modulated radiotherapy for tumors of the nasal cavity and paranasal sinuses: clinical outcomes and patterns of failure. Int J Radiat Oncol Biol Phys. 2012:83(1):243-51.

3. Daly ME, Chen AM, Bucci MK, El-Sayed I, Xia P, Kaplan MJ, et al. Intensitymodulated radiation therapy for malignancies of the nasal cavity and paranasal sinuses. Int J Radiat Oncol Biol Phys. 2007;67(1):151-7.

4. Madani I, Bonte K, Vakaet L, Boterberg T, De Neve W. Intensity-modulated radiotherapy for sinonasal tumors: Ghent University Hospital update. Int J Radiat Oncol Biol Phys. 2009;73(2):424-32.

5. Dirix P, Vanstraelen B, Jorissen M, Vander Poorten V, Nuyts S. Intensitymodulated radiotherapy for sinonasal cancer: improved outcome compared to conventional radiotherapy. Int J Radiat Oncol Biol Phys. 2010;78(4):998-1004.

6. Abu-Ghanem S, Horowitz G, Abergel A, Yehuda M, Gutfeld O, Carmel NN, et al. Elective neck irradiation versus observation in squamous cell carcinoma of the maxillary sinus with N0 neck: A meta-analysis and review of the literature. Head Neck 2014. Epub ahead of print. doi: 10.1002/hed.23791. PMID: 24913744 
7. Mirghani H, Hartl D, Mortuaire G, Armas GL, Auperin A, Chevalier D, et al. Nodal recurrence of sinonasal cancer: does the risk of cervical relapse justify a prophylactic neck treatment? Oral Oncol. 2013;49(4):374-80.

8. Hoppe BS, Stegman LD, Zelefsky MJ, Rosenzweig KE, Wolden SL, Patel SG, et al. Treatment of nasal cavity and paranasal sinus cancer with modern radiotherapy techniques in the postoperative setting-the MSKCC experience. Int J Radiat Oncol Biol Phys. 2007;67(3):691-702.

9. Edge S, Byrd DR, Compton CC, Fritz AG, Greene FL, Trotti A. (ed.): AJCC cancer staging manual. Springer Verlag, New York, USA: 7th edn: Springer; 2010.

10. Wambersie A LT. ICRU Report 62: Prescribing, Recording and Reporting Photon beam Therapy. International Commission on Radiation Units and Measurements, Bethesda, USA: (supplement to ICRU Report 50). 1999.

11. Jiang GL, Ang KK, Peters $L$, Wendt CD, Oswald MJ, Goepfert H. Maxillary sinus carcinomas: natural history and results of postoperative radiotherapy. Radiother Oncol. 1991;21(3):193-200.

12. Katz TS, Mendenhall WM, Morris CG, Amdur RJ, Hinerman RW, Villaret DB. Malignant tumors of the nasal cavity and paranasal sinuses. Head Neck. 2002;24(9):821-9.

13. Tiwari R, Hardillo JA, Tobi H, Mehta D, Karim AB, Snow G. Carcinoma of the ethmoid: results of treatment with conventional surgery and post-operative radiotherapy. Eur J Surg Oncol. 1999;25(4):401-5.

14. Dirix P, Nuyts $S$, Geussens $Y$, Jorissen $M$, Vander Poorten V, Fossion E, et al. Malignancies of the nasal cavity and paranasal sinuses: long-term outcome with conventional or three-dimensional conformal radiotherapy. Int J Radiat Oncol Biol Phys. 2007;69(4):1042-50.

15. Roa WH, Hazuka MB, Sandler HM, Martel MK, Thornton AF, Turrisi AT, et al. Results of primary and adjuvant CT-based 3-dimensional radiotherapy for malignant tumors of the paranasal sinuses. Int J Radiat Oncol Biol Phys. 1994;28(4):857-65.

16. Padovani L, Pommier P, Clippe SS, Martel-Lafay I, Malet C, Poupart M, et al. Three-dimensional conformal radiotherapy for paranasal sinus carcinoma: clinical results for 25 patients. Int J Radiat Oncol Biol Phys. 2003;56(1):169-76

17. Claus F, De Gersem W, De Wagter C, Van Severen R, Vanhoutte I, Duthoy W, et al. An implementation strategy for IMRT of ethmoid sinus cancer with bilateral sparing of the optic pathways. Int J Radiat Oncol Biol Phys. 2001;51 (2):318-31.

18. Chen AM, Daly ME, Bucci MK, Xia P, Akazawa C, Quivey JM, et al. Carcinomas of the paranasal sinuses and nasal cavity treated with radiotherapy at a single institution over five decades: are we making improvement? Int J Radiat Oncol Biol Phys. 2007;69(1):141-7.

19. O'Sullivan B, Rumble RB, Warde P. Intensity-modulated radiotherapy in the treatment of head and neck cancer. Clin Oncol (R Coll Radiol). 2012;24(7):474-87.

20. Jansen EP, Keus RB, Hilgers FJ, Haas RL, Tan IB, Bartelink H. Does the combination of radiotherapy and debulking surgery favor survival in paranasal sinus carcinoma? Int J Radiat Oncol Biol Phys. 2000;48(1):27-35.

21. Bentz BG, Bilsky MH, Shah JP, Kraus D. Anterior skull base surgery for malignant tumors: a multivariate analysis of 27 years of experience. Head Neck. 2003;25(7):515-20

22. Suh YG, Lee CG, Kim H, Choi EC, Kim SH, Kim CH, et al. Treatment outcomes of intensity-modulated radiotherapy versus three-dimensional conformal radiotherapy for patients with maxillary sinus cancer in the postoperative setting. Head Neck 2014. Epub ahead of print. doi: 10.1002/hed.23791. PMID: 24913744

23. Pignon JP, le Maitre A, Maillard E, Bourhis J. Meta-analysis of chemotherapy in head and neck cancer (MACH-NC): an update on 93 randomised trials and 17,346 patients. Radiother Oncol. 2009;92(1):4-14.

24. Airoldi M, Garzaro M, Valente G, Mamo C, Bena A, Giordano C, et al. Clinical and biological prognostic factors in 179 cases with sinonasal carcinoma treated in the Italian Piedmont region. Oncology. 2009;76(4):262-9.

25. Myers LL, Nussenbaum B, Bradford CR, Teknos TN, Esclamado RM, Wolf GT. Paranasal sinus malignancies: an 18-year single institution experience. Laryngoscope. 2002;112(11):1964-9.

26. Pfister DG, Ang KK, Brizel DM, Burtness BA, Busse PM, Caudell JJ, et al. Head and neck cancers, version 2.2013. Featured updates to the NCCN quidelines. J Natl Compr Canc Netw. 2013;11(8):917-23.

27. Takeda A, Shigematsu N, Suzuki S, Fujii M, Kawata T, Kawaguchi O, et al. Late retinal complications of radiation therapy for nasal and paranasal malignancies: relationship between irradiated-dose area and severity. Int J Radiat Oncol Biol Phys. 1999;44(3):599-605.

\section{Submit your next manuscript to BioMed Central and take full advantage of:}

- Convenient online submission

- Thorough peer review

- No space constraints or color figure charges

- Immediate publication on acceptance

- Inclusion in PubMed, CAS, Scopus and Google Scholar

- Research which is freely available for redistribution

Submit your manuscript at www.biomedcentral.com/submit 